Cultures \& Conflits

72 | hiver 2008

Frontières et logiques de passage

\title{
Au sujet de Los Nietos, un film de Marie-Paule Jeunehomme
}

\section{Marie-Paule Jeunehomme}

\section{(2) OpenEdition \\ 1 Journals}

\section{Édition électronique}

URL : http://journals.openedition.org/conflits/14203

DOI : $10.4000 /$ conflits. 14203

ISSN : $1777-5345$

Éditeur :

CCLS - Centre d'études sur les conflits lilberté et sécurité, L'Harmattan

\section{Édition imprimée}

Date de publication : 20 décembre 2008

ISBN : 1157-966X

ISSN : 1157-996X

\section{Référence électronique}

Marie-Paule Jeunehomme, «Au sujet de Los Nietos, un film de Marie-Paule Jeunehomme », Cultures \& Conflits [En ligne], 72 I hiver 2008, mis en ligne le 15 janvier 2009, consulté le 30 mars 2021. URL :

http://journals.openedition.org/conflits/14203 ; DOI : https://doi.org/10.4000/conflits.14203

Ce document a été généré automatiquement le 30 mars 2021.

Creative Commons License 


\title{
Au sujet de Los Nietos, un film de Marie-Paule Jeunehomme
}

\author{
Marie-Paule Jeunehomme
}

La réalisatrice belge Marie-Paule Jeunehomme a récemment consacré un documentaire à la question des exhumations en Espagne. Le film appelé Los Nietos ${ }^{1}$ (les petits-enfants) se passe à San Pedro Mallo, un petit village du Bierzo où il existe une fosse clandestine, renfermant un corps, celui de Leonides Rodríguez. C'est lui que l'on cherche et, ce faisant, la réalisatrice recueille la parole de ceux qui, à un titre ou à un autre, se sentent concernés par ce travail. Nous reproduisons ci-après un bref entretien ainsi que quelques extraits de dialogues issus du film ${ }^{2}$.

C\&C : Pourquoi un film sur les petits-enfants ?

M.-P. J :Au départ, ce qui m'a interpellée, c'est que ce sont des nietos qui ont entrepris de réaliser ces exhumations. C'est Emilio [Silva] sur les traces de son grand-père qui a ouvert la voie. Pourquoi tout d'un coup cette histoire refoulée, intime, pouvait-elle reprendre vigueur, au point de s'exprimer publiquement? Pourquoi si tard? On sait par exemple que, pour la Shoah, un phénomène identique s'est produit. Souvent, les rescapés n'ont pu parler qu'à partir du moment où leurs petits-enfants ont pu entendre. Ici, en Espagne, on est sans doute dans le même schéma, avec en plus, une donnée historique qui est la durée de la dictature, près de quarante ans, dictature qui a imposé le silence. Et puis, dans ces petits villages, les familles de victimes et les familles de bourreaux ont continué à vivre côte à côte. J'ai été frappée par la longueur de ce silence. J'ai été frappée par le courage de ces nietos à affronter un passé enfoui, à imposer le retour de la parole.

Lorsque j'ai travaillé sur ce thème pour un reportage radio (deux ans avant le film), j'ai rencontré des nietos qui avaient entrepris ce travail de mémoire, d'exhumation de leurs proches. J'ai pu approcher leur ressenti, ce qu'était leur quête, leurs espoirs. C'est cela que j'ai voulu donner à voir dans ce film. L'écrivain espagnol Manuel Rivas, au cours de ce reportage radio (je l'avais alors longuement interviewé), avait eu des mots que je te reproduis. Ils ont servi de fil conducteur au cheminement de ma propre pensée, de mes envies par rapport au film. 
" Après la dictature, il y a eu une démocratie sous surveillance, assombrie. Il y a eu comme un divorce entre la mémoire privée, intime, émotionnelle et je dirais même secrète des gens, et la mémoire officielle, présentée comme la normalité. Mais je dirais que cette "normalité" est anormale et immorale. C'est une des raisons pour lesquelles j'écris, c'est un peu comme un processus de guérison [...]. C'est terrible de voir que les plus beaux sites de Galice sont des lieux où des crimes ont été commis. C'est comme si on avait occulté le paysage. Les paysages du temps et de la vie. Occulté avec un rideau. L'immoralité consistant à se taire se transmet comme une maladie. Il arrive un moment où tu te rends compte qu'il est nécessaire de lever le rideau. C'est le premier pas pour que la société entière ne soit pas contaminée par cette maladie, cette suspension de la conscience ».

C'est cette suspension de la conscience que je voulais donner à voir. Et comment aujourd'hui le processus de guérison s'est mis en marche. J'aime l'histoire d'Espagne, je l'ai étudiée, épluchée, mais c'est surtout la façon dont elle se réécrit aujourd'hui qui m'intéresse. Qui, pourquoi, comment, avec quelles douleurs et quels soulagements. Les nietos, c'est nous, c'est notre génération. Je voulais être dans le présent. Je ne voulais pas écrire une page d'histoire, avec archives en noir et blanc, je voulais parler de cette mémoire en mouvement, cette mémoire qui est notre fondement à chacun. L'occulter, la nier, c'est nous amputer. Ce que montre le film se passe aujourd'hui dans toute l'Espagne, au bord des routes, des champs, à la lisière des cimetières, partout des mains creusent pour que l'oubli ne recouvre pas tout, pour que cette conscience humaine ne s'efface pas à jamais. Qu'elle reste présente dans les paysages. J'avais envie que les spectateurs qui iront se promener en Espagne après avoir vu le film, voient les paysages autrement.

J'avais aussi envie de partir de l'intime, de la quête d'une personne, ici Mari Carmen, qui cherche son grand-père (Leonides Rodríguez), voir ce qui se produisait pour elle et ses proches une fois la parole libérée, une fois le corps de son grand-père retrouvé. Je voulais être dans le ressenti, pas dans l'information. Je ne voulais pas faire un cours, mais simplement suivre ce cheminement intime d'une mémoire, volée, qui se réapproprie sa place. Et voir comment cette mémoire intime dévoilait la mémoire d'une histoire collective.

« Nous sommes en grande partie ce dont nous nous souvenons. La mémoire nous permet précisément de voir les lieux. On se trouve parfois face à des paysages et il nous semble qu'on est en train de voir, et nous ne voyons pas. Comme si c'était couvert par le brouillard. Quand on connaît cette histoire, les récits, ce qui s'est passé là, on voit de nouveau. Il y a cette beauté géographique et en même temps la force d'une histoire humaine, d'une vérité humaine, enterrée ici. Et c'est comme si tu redécouvrais ton pays. "

Manuel Rivas, écrivain ${ }^{3}$

«Quand la première exhumation a été montrée à la télévision, les gens ont réalisé que c'était possible. Ils savent qu'une fois la dépouille trouvée, la science peut déterminer s'il s'agit d'un de leurs proches. Et surtout que ça ne prête pas à conséquence, que c'est normal. Ce n'est pas normal qu'ils gisent dans une fosse, d'où notre travail. Même si, quarante après, les gens ont encore peur. Je me rappelle une exhumation [...]. Un des fils des victimes qu'on exhumait disait que les phalangistes ${ }^{4}$ allaient venir nous tuer tous. Mais le message social le plus important, c'est qu'il ne s'agissait pas de rouges, de meurtriers, de brûleurs d'églises. C'était les républicains, les démocrates, vainqueurs des élections, ceux qui gouvernaient démocratiquement le pays. Ceux qui avaient créé des milliers d'écoles dans tout le pays. Ceux qui avaient misé sur la culture [...]. »

Propos de Santiago Macías, l'un des fondateurs de l'ARMH ${ }^{5}$ 
«On nous demande souvent pourquoi ce sont les petits-enfants qui font ce travail. La réponse est facile. L'Espagne a vécu sous une dictature pendant quarante ans. Les familles qui ont été victimes de la répression n'ont rien dit à leurs enfants pour les protéger. Afin de les épargner. Ces enfants-là sont nos parents. Notre génération, en quelque sorte, s'est libérée de cette peur. Et nous voulons savoir ce qui s'est passé. Nous voulons rendre leur dignité à ces personnes qui sont mortes et gisent dans des fosses communes [...]. Cela peut sembler un peu tard mais mieux vaut tard que jamais. »

Javier Ortiz, archéologue, membre de l'ARMH

«Ça s'est passé il y a si longtemps. Nous, les petits-enfants, ignorons parfois ce que nos grands-parents ont fait. On ne s'en souvient pas. Ou on ne nous le dit pas. Parfois, il nous manque des informations sur qui étaient nos aïeux. Ce qu'ils ont fait [...]. Quoi qu'il en soit, quel que soit le passé de nos grands-parents, ces gens méritent d'être exhumés. »

José Luis González, habitant de San Pedro, engagé auprès de l'ARMH

C\&C : Dans nos échanges informels, tu as évoqué le mot « réconciliation ». Je dois t'avouer que ce mot me dérange. Au cours de mes propres recherches sur la manière dont la question des «disparus » a été abordée au Chili par les hommes politiques, en particulier dans les années 1990, il m'est apparu que ce terme était là pour nommer une politique d'Etat qui a systématiquement dénié aux familles des « disparus » l'une de leurs principales revendications (justice). Je comprends que tu donnes à ce terme un sens différent. Je comprends que tu fais allusion à une coexistence au jour le jour, dans un espace clairement délimité, des familles des fusillés et des familles de ceux qui ont tué ou dénoncé, notamment dans les villages comme San Pedro. Une coexistence qui s'est poursuivie dans le temps de telle sorte qu'aujourd'hui vivent dans un même village les petits-enfants d'un camp et de l'autre. Je voudrais que cet entretien garde une trace, sans donner de nom bien entendu, de cette histoire que tu m'as racontée au cours de nos premières conversations, au sujet d'un homme qui cherche quelqu'un qui n'est pas un de ses proches...

M.-P. J :J'ai mis un peu de temps à te répondre, mais c'est que ta question me «turlupine ", si tu comprends ce que cela veut dire... La réconciliation ? Dans le cas de San Pedro en tout cas, quelque chose s'est passé à la suite de cette exhumation du grand-père de Mari Carmen et après les fouilles, sans succès, du deuxième corps. Je vais tenter de te le décrire. Au début du tournage, Mari Carmen refusait de se faire interviewer dans le village, elle ne voulait pas que les gens du village entendent ce qu'elle allait nous raconter. Elle avait peur que l'exhumation soit mal perçue par une partie du village. Même chez les trois vieilles personnes, il y avait de la méfiance, on le voit dans le film, quand deux d'entre elles se retournent et disent: "regarde qui vient là pour voir ce que l'on est en train de faire... Qui est-ce? ». Une autre fois, alors que l'on interviewait une de ces trois petites vieilles, elle a brusquement changé de conversation au milieu d'une phrase, en nous faisant signe des yeux... Quelqu'un s'était approché derrière nous, et cette vieille ne voulait pas qu'on sache ni entende de quoi elle nous parlait.

Ces malaises petit à petit se sont éteints. Mari Carmen a commencé à parler à P.G. ${ }^{6}$, elle est venue partager un déjeuner avec nous et les bénévoles chez lui. Elle lui était reconnaissante d'être venu voir l'exhumation de son grand-père, d'avoir reconnu que son grand-père à lui était sans doute pour quelque chose dans la dénonciation et donc dans la mort du sien. "On peut maintenant se regarder dans les yeux" nous a-t-elle dit, " regardant non plus vers le passé, mais ensemble vers l'avenir pour que ce genre de choses ne se reproduise pas ». A notre départ, au bout d'un mois, tout le monde était là pour nous 
dire au revoir, alors que quand nous sommes arrivés, une partie du village restait distante.

Ce n'est peut-être pas une réconciliation, mais une page nouvelle s'est écrite dans le village. A regarder le passé ensemble à travers les fouilles (en participant, comme P.G. 7, ou en étant présents pour regarder), les villageois se sont retrouvés sur cette nécessité de rendre les corps à leurs familles. Cela leur a permis de vivre quelque chose de fort ensemble (succès d'une exhumation, échec de l'autre). Quelque part, ils ont affronté le passé, ils l'ont accepté pour ce qu'il a été, sans plus le cacher, et ils ont pu le dépasser. Cette histoire a repris sa place dans les conversations, comme l'explique un des villageois à la fin: "j'aime l'ambiance qu'il y a aujourd'hui dans le village, les langues se délient, aujourd'hui on en parle».

Je ne veux pas généraliser au sujet de la réconciliation. Aujourd'hui en Espagne, il y a toujours des personnes (et notamment au Parti populaire, dans l'Eglise) qui refusent toute exhumation, qui mettent des bâtons dans les roues, qui refusent de condamner le franquisme...

\section{NOTES}

1. Los Nietos, 59 minutes, 2008, une production du Centre vidéo de Bruxelles (CVB), Michel Steyaert en coproduction avec la RTBF, le Centre de l'audiovisuel à Bruxelles (CBA) et des Territoires de la mémoire. Le film a également bénéficié de l'aide du Centre du cinéma et de l'audiovisuel de la communauté française de Belgique et des télédistributeurs wallons.

2. Entretien réalisé par Antonia García Castro, à distance (Bruxelles, Buenos Aires) par courrier électronique, en mai 2008. Nous remercions la réalisatrice d'avoir bien voulu nous envoyer son film à Buenos Aires afin de pouvoir réaliser ce bref entretien « en connaissance de cause ».

3. Cet extrait et les suivants sont tirés du film de Marie-Paule Jeunehomme.

4. Membres de la Phalange espagnole, créée en 1933 par Primo de Rivera, parti d'extrême droite.

5. Coauteur de l'ouvrage Les Fosses du franquisme, op. cit.

6. Nous avons volontairement anonymisé le nom de cette personne en raison de la particularité de son itinéraire (évoqué par Marie-Paule Jeunehomme) et de la pudeur qui a été la sienne au moment d'en parler, hors caméra (d'après le récit que nous en a fait la réalisatrice dans nos conversations informelles).

7. Ainsi P.G., dont le grand-père a peut-être dénoncé celui de Mari Carmen, a lui-même participé aux fouilles en vue de localiser une deuxième fosse commune dans ce village (qui n'a pas été retrouvée). 


\section{AUTEUR}

\section{MARIE-PAULE JEUNEHOMME}

Marie-Paule Jeunehomme est née à Verviers (Belgique) en 1959. Elle est licenciée en journalisme et en coopération au développement de l'université libre de Bruxelles. Elle travaille depuis 1986 à la RTBF, radio télévision belge, puis comme journaliste à la rédaction radio depuis 1987. Elle a réalisé en 1996 une série de documentaires radiophoniques sur la Guerre d'Espagne, les Brigades internationales, l'accueil en Belgique des « enfants de la guerre » espagnols, puis en 2006, un documentaire en deux parties de 30 minutes sur le travail de mémoire en Espagne, Les Chemins de la mémoire. Elle a obtenu le Prix Dexia de la presse radiophonique belge en 2005 pour un documentaire : Les Prisons : au-delà des peines. Los Nietos est son premier documentaire pour la télévision en tant que réalisatrice. Il est le fruit d'un long travail sur l'Espagne et la mémoire du franquisme, entrepris il y a près de vingt ans. 